Research Article

\title{
Research on the Operating Efficiency of Chinese Listed Pharmaceutical Companies Based on Two-Stage Network DEA and Malmquist
}

\author{
Tsung-Xian Lin $\left(\mathbb{D},{ }^{1}\right.$ Zhong-huan Wu ${ }^{D},{ }^{1}$ Xiao-xia Ji $\mathbb{D}^{2},{ }^{2}$ and Jia-jia Yang $\mathbb{D}^{3}$ \\ ${ }^{1}$ Department of Business Administration, Guangzhou Huashang College, Guangzhou 511300, China \\ ${ }^{2}$ School of Business, Guangdong University of Foreign Studies, Guangzhou 510000, China \\ ${ }^{3}$ Department of Public Policy, King's College London, London WC2R 2LS, UK
}

Correspondence should be addressed to Xiao-xia Ji; 976224056@qq.com

Received 20 May 2021; Accepted 16 June 2021; Published 23 June 2021

Academic Editor: Yiwen Zhang

Copyright (c) 2021 Tsung-Xian Lin et al. This is an open access article distributed under the Creative Commons Attribution License, which permits unrestricted use, distribution, and reproduction in any medium, provided the original work is properly cited.

\begin{abstract}
The development of pharmaceutical companies, which is an important part of the national economy and industry, is closely related to people's livelihood issues. With the era of big data, this paper uses the two-stage DEA and Malmquist method to evaluate the efficiency of listed Chinese pharmaceutical companies. From a static and dynamic perspective, it analyses the total factor productivity index, pure efficiency change index, scale efficiency index, and so on. The results show that government subsidies have not had a positive impact on most Chinese pharmaceutical companies, and for films, diseconomies of scale caused by rapid expansion should be avoided.
\end{abstract}

\section{Introduction}

Since the outbreak of the COVID-19, medical health has become the primary topic of concern for the government and the public. The operation of pharmaceutical companies has become the focus of discussion. At present, China's pharmaceutical manufacturing industry has long been in a state of low industry concentration, high product homogeneity, and weak technological innovation capabilities. It has a large gap with the international advanced level. In recent years, China's various medical reform policies and regulations have been promulgated one after another. It has brought many uncertain factors to the development of the pharmaceutical industry. How to adapt to changes in the external environment and further improve operating efficiency has become an important issue facing the government and enterprises. Based on the above background, this article focuses on the research on the operating efficiency of pharmaceutical companies, innovatively combining the DEA method with Malmquist, taking 164 listed companies in China as a sample, and studying their operating conditions during the five-year period from 2015 to 2019.

The structure of the following parts of this article is as follows. Section 2 collects current scholars' research on the efficiency evaluation of pharmaceutical companies; Section 3 introduces the related content of the two-stage network DEA and Malmquist index method in this article; Section 4 outlines the research of this article process, sample selection, and two-stage DEA data analysis. Section 5 uses Malmquist to analyse the dynamic effects of the data and finally summarizes the research results of this article. 


\section{Literature Review}

At present, many scholars use the DEA method to measure the actual situation of the operating efficiency of pharmaceutical companies and provide suggestions for improvement of the company's operations. For example, Wang [1] selected Chinese biopharmaceutical companies from 2017 to 2019 as a research sample and established a static DEA model to measure their financing efficiency. The results show that although the overall financing efficiency of Chinese biotech companies is not high, the level of it is increasing year by year. And Zhang Zicheng [2] innovatively combined the AHP method with the DEA method, finding that, compared with the scale factor, the deficiency of technology hinders the operating performance of companies such as Lunan Pharmaceutical firm. Meanwhile, Li et al. [3] used a two-step method of factor analysis and SE-DEA model to calculate the financial data of 58 listed pharmaceutical companies in China from 2009 to 2013 and concluded that the overall inefficiency of the pharmaceutical industry is also due to insufficient investment on scale and technology.

Among them, some scholars even divide medical companies into groups to study their operational status in different regions $[4,5]$. The research found that there are indeed differences in the operating efficiency as well as in terms of technological innovation of pharmaceutical companies in different regions [6, 7]. For example, Xiong [8] used the panel data of technological innovation of medical companies listed in Guangdong, Shandong, Zhejiang, and Jiangsu as samples to study the allocation of technological innovation resources of pharmaceutical manufacturers in four provinces in China from 2015 to 2017 and finally came to the following conclusions: in the province, Jiangsu pharmaceutical companies have the advantage of pure technical efficiency in innovation activities, while Guangdong pharmaceutical companies performed better in scale efficiency with regard to technological innovation. However, the traditional DEA model cannot study the influencing factors in the process. Therefore, the research studies on the two-stage network DEA model and the Malmquist index method have received widespread attention from many scholars.

Regarding the two-stage network DEA, such method has been applied to plenty of fields. For example, Lewis et al. applied the undirected network DEA method to the efficiency evaluation of Major League Baseball [9]. Additionally, Liang et al. used a two-stage network DEA model to analyse the input-output efficiency of 50 universities in China [10], while $\mathrm{Li}$ et al. applied the DEA model under the two-stage expansion structure to the research on the efficiency of R\&D in China's provincial regions [11]. At the same time, some researchers also combine two-stage network DEA with other methods. For instance, Chen et al. [12] and Kao [13] combined it with the two-stage additive efficiency decomposition DEA method to study the relative efficiency of 24 non-life insurance companies in Taiwan. Lee and Johnson combined Malmquist under the network DEA structure to study the performance of the semiconductor manufacturing industry [14]. It can be seen that the two-stage network DEA has been widely used in insurance companies, universities, and other industry research. However, research in the pharmaceutical industry is still relatively rare.

Regarding the Malmquist index method, scholars have also made great achievements. In the field of sustainable e-agriculture, Pan and others used the 31 provinces as the research objects and explored the sustainable development efficiency of agriculture in mainland China through DEA and Malmquist productivity index models [15]. In the medical industry, Hashimoto and Haneda [16] used the conventional DEA method and the Malmquist productivity index method to measure the R\&D efficiency of the Japanese pharmaceutical industry from the enterprise level and the industry level, respectively. Empirical evidence shows that the total factor productivity of Japan's pharmaceutical industry is declining, and the main reason for the decline is the sharp decrease in technological changes. What is more, Pannu et al. [17] used the output-oriented VRS model and the Malmquist productivity index method to measure the increase in efficiency and productivity of the Indian pharmaceutical industry over a 10-year period, finding that the increase was mainly due to the growths in technical efficiency. Furthermore, Zhiyue and Qiu [18] also used the Malmquist index method to conduct an empirical analysis of the operating efficiency of China's biopharmaceutical industry from both horizontal and vertical aspects. The results show that the overall operating efficiency of the biopharmaceutical industry is not ideal, and there is a large difference in efficiency between provinces and cities.

In summary, it can be seen that scholars have used many different methods to study the operating efficiency of pharmaceutical manufacturing enterprises, but the research still has the following shortcomings. Firstly, most research studies on the efficiency of pharmaceutical manufacturing enterprises use nonparametric methods. When measuring enterprise efficiency, some scholars only consider a certain aspect of static or dynamic and thus cannot comprehensively analyse the efficiency level and development trend of pharmaceutical manufacturing enterprises. Secondly, there are few literatures on the research of listed pharmaceutical companies using the two-stage network DEA and Malmquist index method, most of which focus on the traditional DEA method. Finally, in the literature on efficiency influencing factors, the selection of variables is relatively limited, and there are few literatures that consider the R\&D capabilities of enterprises. For pharmaceutical manufacturing companies, environmental variables are very important and have a very large impact on the efficiency of the company. Therefore, the external environment of the company should be considered when studying the efficiency of the company. Based on the above deficiencies, this paper uses the two-stage network DEA and Malmquist index method to study the operating efficiency of enterprises from both static and dynamic perspectives. When studying the factors affecting the operating efficiency of enterprises, environmental variables have been added and considered from multiple angles in the article, striving for a more comprehensive selection of influencing factors. 


\section{Research Method}

3.1. Two-Stage Network DEA. In the traditional DEA model, we only know the final efficiency values of the entire process, but the specific situation in the whole process is unknown. The information provided by the traditional DEA model is not enough, and the guidance to managers is limited. The two-stage network DEA model can open the "black box" of the production system, which can effectively measure the complex production network. Therefore, this paper also adopts the two-stage network DEA model for performance evaluation and pays more attention to the progressive relationship between the two stages based on the research results of other scholars. Its internal structure is shown in Figure 1.

Among them, $X_{i j}^{1}(i=1,2, \ldots, I)$ represents the $i$-th input of $\mathrm{DMU}_{j}$ in the first stage; $Z_{d j}(d=1,2, \ldots, D)$ represents the intermediate variable, namely, it is not only the $d$-th output of $\mathrm{DMU}_{j}$ in the first stage, but also the $d$-th input of $\mathrm{DMU}_{\mathrm{j}}$ in the second stage; $X_{k j}^{2}(k=1,2, \ldots, K)$ represents the $k$-th input of the newly added $\mathrm{DMU}_{j}$ in the second stage; and $y_{r j}(r=1,2, \ldots, R)$ represents the $r$-th output of $\mathrm{DMU}_{j}$ in the second stage. First of all, calculate the efficiency of the first stage and then calculate the efficiency of the second stage on this basis, which means keeping the efficiency of the first stage unchanged. Finally, the product of the efficiency of the two stages is regarded as the total efficiency of the system. At this point, the model can be established as follows:

The first-stage model (model 1) is given by

$$
\begin{gathered}
\operatorname{Max} \frac{\sum_{d=1}^{D} W_{d} Z_{d 0}}{\sum_{i=1}^{I} V_{i} X_{i 0}^{1}}, \\
\text { s.t. } \frac{\sum_{d=1}^{D} W_{d} Z_{d j}}{\sum_{i=1}^{I} V_{i} X_{i j}^{1}} \leq 1, \quad j=1,2, \ldots, n, \\
\frac{\sum_{r=1}^{R} U_{r} Y_{r j}}{\sum_{d=1}^{D} z_{d j}+\sum_{k=1}^{k} t_{k} x_{k j}^{2}} \leq 1, \quad j=1,2, \ldots, n, \\
U_{r}, V_{i}, W_{d}, t_{k} \geq 0, \quad \forall r, i, d, k .
\end{gathered}
$$

Model 1 adds a constraint on the basis of the traditional CCR model, that is, the last constraint. Its purpose is to ensure that the optimal solution of the first stage makes the efficiency value of the second stage not more than 1 , so as to ensure that the second stage model must have a feasible solution; otherwise, there may be no feasible solution. Therefore, this constraint is necessary, which was not considered in the previous two-stage DEA model. In model $1, U_{r}, V_{i}, W_{d}, t_{k}$, respectively, represent the weights of the corresponding variables, after considering the study conducted by Cheng and Zheng [19]. The efficiency of each DMU can be obtained by model 1. Record the efficiency of the $\mathrm{DMU}_{0}$ as $\theta_{1}^{0 *}$. Then second-stage model (model 2) is given by

$$
\begin{gathered}
\operatorname{Max} \frac{\sum_{r=1}^{R} U_{r} Y_{r 0}}{\sum_{d=1}^{D} W_{d} Z_{d 0}+\sum_{k=1}^{k} t_{k} x_{k 0}^{2}}, \\
\text { s.t } \frac{\sum_{d=1}^{D} W_{d} Z_{d j}}{\sum_{i=1}^{I} V_{i} X_{i j}^{1}} \leq 1, j=1,2, \ldots, n, \\
\frac{\sum_{r=1}^{R} U_{r} Y_{r j}}{\sum_{d=1}^{D} W_{d} Z_{d j}+\sum_{k=1}^{k} t_{k} x_{k j}^{2}} \leq 1, \quad j=1,2, \ldots, n, \\
\frac{\sum_{d=1}^{D} W_{d} Z_{d 0}}{\sum_{i=1}^{I} V_{i} X_{i 0}^{1}}=\theta_{1}^{0 *}, \\
U_{r}, V_{i}, W_{d}, t_{k} \geq 0, \quad \forall r, i, d, k .
\end{gathered}
$$

Solving (5)-(9) can get the efficiency of the second-stage DMU, noting $\theta_{2}^{0 *}$ as the efficiency of the second stage. So far, it can be concluded that the total efficiency of the two-stage system $\mathrm{DMU}_{0}$ is $\theta^{0 *}=\theta_{1}^{0 *} \theta_{2}^{0 *}(10)$.

3.2. Malmquist Index. The two-stage network DEA model just horizontally compared the efficiency of listed pharmaceutical enterprises. So we will build the Malmquist index model to make a longitudinal analysis of efficiency and dynamically analyse the change of efficiency.

TFP is total factor productivity index:

$$
\begin{aligned}
\operatorname{TFP}= & \frac{D_{t}^{u}\left(x_{t}, y_{t}\right)}{D_{t-1}^{u}\left(x_{t-1}, y_{t-1}\right)} \times\left[\frac{\left(D_{t}\left(x_{t}, y_{t}\right) / D_{t-1}^{C}\left(x_{t-1}, y_{t-1}\right)\right)}{\left(D_{t}^{u}\left(x_{t}, y_{t}\right) / D_{t-1}^{u}\left(x_{t-1}, y_{t-1}\right)\right)}\right] \\
& \times\left[\frac{D_{t-1}^{C}\left(x_{t-1}, y_{t-1}\right)}{D_{t}^{C}\left(x_{t-1}, y_{t-1}\right)} \times \frac{D_{t-1}^{C}\left(x_{t}, y_{t}\right)}{D_{t}^{C}\left(x_{t}, y_{t}\right)}\right]^{(1 / 2)} .
\end{aligned}
$$

PEC is pure efficiency change index:

$$
\mathrm{PEC}=\frac{D_{t}^{u}\left(x_{t}, y_{t}\right)}{D_{t-1}^{u}\left(x_{t-1}, y_{t-1}\right)},
$$

SE is scale efficiency index:

$$
\mathrm{SE}=\frac{\left(D_{t}^{C}\left(x_{t}, y_{t}\right) / D_{t-1}^{C}\left(x_{t-1}, y_{t-1}\right)\right)}{\left(D_{t}^{4}\left(x_{t}, y_{t}\right) / D_{t-1}^{u}\left(x_{t}-1, y t-1\right)\right)},
$$

TC is technical change index:

$$
\mathrm{TC}=\left[\frac{D_{t-1}^{C}\left(x_{t-1}, y_{t-1}\right)}{D_{t}^{C}\left(x_{t-1}, y_{t-1}\right)} \times \frac{D_{t-1}^{C}\left(x_{t}, y_{t}\right)}{D_{t}^{C}\left(x_{t}, y_{t}\right)}\right]^{(1 / 2)},
$$

The formula of total factor productivity is

$$
\mathrm{TFP}=\mathrm{PEC} \times \mathrm{SE} \times \mathrm{TC} .
$$




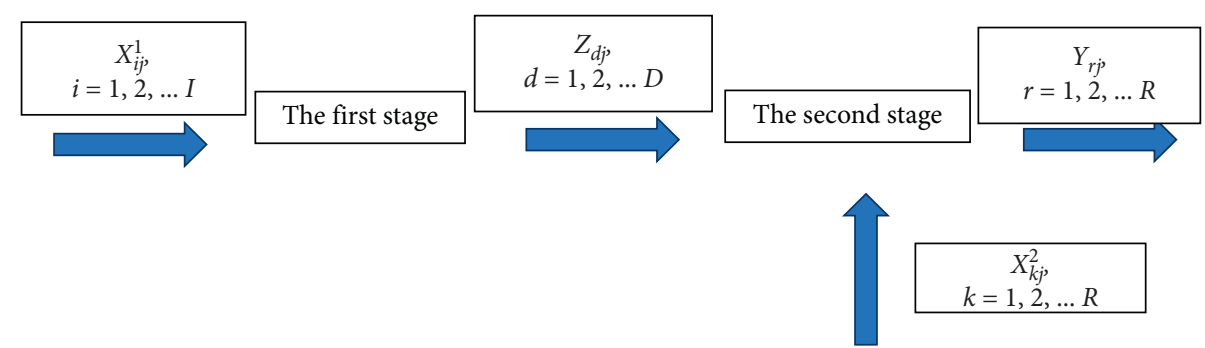

Figure 1: The two-stage network DEA model.

TABLE 1: Variable selection and measurement.

\begin{tabular}{|c|c|c|c|}
\hline & Variable type & Variable name & Measure \\
\hline The first stage & Output & $\begin{array}{l}\text { Gross costs } \\
\text { Total number of employees } \\
\text { Net value of fixed assets } \\
\text { Gross revenue }\end{array}$ & $\begin{array}{c}\text { Gross costs in corporate annual reports } \\
\text { Total number of in-service staff } \\
\text { Net value of fixed assets in corporate annual reports } \\
\text { Gross revenue in corporate annual reports }\end{array}$ \\
\hline The second stage & $\begin{array}{l}\text { Output } \\
\text { Input }\end{array}$ & $\begin{array}{l}\text { Net profit } \\
\text { Gross costs } \\
\text { Government subsidies }\end{array}$ & $\begin{array}{l}\text { Total profit }- \text { income tax } \\
\text { Government subsidies that are included in the current profit and loss }\end{array}$ \\
\hline
\end{tabular}

\section{Empirical Analysis}

4.1. Sample Selection. With reference to the definition of pharmaceutical companies, combined with the description of the main business in the annual report of the A-share listed pharmaceutical company and the proportion of the main business in operating income, the study sample is determined. At the same time, to ensure the validity of the analysis results, ST, PT, and ST* companies were excluded, and 164 listed pharmaceutical companies were finally identified as the research samples. The data of inputs and outputs comes from Cathay Pacific database and the annual public report of enterprise.

\subsection{Variable Selection}

4.2.1. The First Stage of Input and Output Variables. The selection of variables is based on character of listed pharmaceutical companies, and we fully inspect the business characteristics and operation of listed pharmaceutical companies. The inputs selected are gross costs $\left(X_{1}\right)$, total number of employees $\left(X_{2}\right)$, and net value of fixed assets $\left(X_{3}\right)$, and the output is gross revenue $\left(Y_{1}\right)$.

4.2.2. The Second Phase of Input and Output Variables. This paper comprehensively examines the business characteristics and operation of listed pharmaceutical companies. Government subsidies $\left(X_{5}\right)$ as a new input are added to the second stage, and the other input is the gross revenue $\left(X_{4}\right)$ that is the output of first stage, and the final output is net profit $\left(Y_{2}\right)$. More details are shown in Table 1 .

4.3. DEA Efficiency Analysis in the First Stage. In first stage (shown in Table 2), no listed pharmaceutical company's technical efficiency reached 1 during 2015-2019. There are 119 companies with technical efficiency between 0.6 and 1.0, accounting for $73 \%$, indicating that the technical efficiency of these companies is good. The efficiency values of the eight securities companies (Chongqing Taiji Industry, Baiyunshan, Kelun, Huabei, Hisun, Harbin, China Medicine, and Renfu) are below 0.4, indicating that the technical efficiency level of these companies is relatively low, and they need to increase investment.

Five securities companies, Adisseo, Changchun HighTech, Hualan Biological Engineering, Hengrui, and Zhifei, have achieved technology effectiveness in some years. Adisseo's technical efficiency is effective in 2016 and has dropped significantly after 2016, and the technical efficiency can be improved by referring to the operation method of 2016 when the technology is effective (see Table 3 for specific annual data).

4.4. DEA Efficiency Analysis in the Second Stage. In the second stage (shown in Table 2), there is no company that has reached technical efficiency of 1 during 2015-2019, indicating that all the companies were not effective. There are 97 companies with a technical efficiency value of 0.6-1.0, accounting for $59.5 \%$, which is significantly lower than the first stage. Only 3 companies had technical efficiency below 0.4 (Sinopharm, Xinbang, and Baiyunshan). The technical efficiency in second stage is generally low, but companies with lower efficiency have been promoted, which may be correlated with government subsidies.

Adisseo, Dezhan Health, Jiao, Hengrui, Livzon Group, Shanghai RAAS, and Zhifei Biotechnology have achieved technical efficiency of 1 in some years. And Adisseo is the same as the first stage, the technical efficiency is effective in 2016 and has dropped dramatically after 2016. The technical efficiency of Dezhan Health and Jiao pharmaceutical companies in the second stage has increased; we believe that the first stage is relevant to the second stage in these companies. However, the average technical efficiency of Hualan 
TABLE 2: Summary of means of technical efficiency.

\begin{tabular}{|c|c|c|c|}
\hline Company name & The first stage & The second stage & Total \\
\hline Adisseo & 0.731807447 & 0.70427645 & 0.718041949 \\
\hline Anke Bio & 0.701258545 & 0.65681619 & 0.679037367 \\
\hline Osaikon & 0.77517144 & 0.677756486 & 0.726463963 \\
\hline Baiyunshan & 0.383760611 & 0.329568568 & 0.35666459 \\
\hline Bdyy & 0.621063253 & 0.668172657 & 0.644617955 \\
\hline Beilu & 0.755456729 & 0.662521895 & 0.708989312 \\
\hline Porton & 0.626443198 & 0.653894142 & 0.64016867 \\
\hline None & 0.708205489 & 0.644792119 & 0.676498804 \\
\hline Changchun High-Tech & 0.754140117 & 0.760920762 & 0.757530439 \\
\hline Changjiang Runfa Medicine & 0.622444299 & 0.581956694 & 0.602200497 \\
\hline Changshan Pharma & 0.705379957 & 0.663215003 & 0.68429748 \\
\hline DAJY & 0.611416622 & 0.570090491 & 0.590753556 \\
\hline Dezhan & 0.800138709 & 0.776221753 & 0.788180231 \\
\hline Jiao & 0.68813071 & 0.761262056 & 0.724696383 \\
\hline DBBT & 0.715738024 & 0.641593159 & 0.678665591 \\
\hline $\mathrm{VC}$ & 0.463121349 & 0.46703195 & 0.465076649 \\
\hline Dongcheng & 0.67384118 & 0.680702372 & 0.677271776 \\
\hline Nhwa Pharm & 0.655734649 & 0.667349718 & 0.661542183 \\
\hline Ekzy & 0.679041427 & 0.578708106 & 0.628874767 \\
\hline Fangsheng & 0.700194471 & 0.656633368 & 0.678413919 \\
\hline Fengyuan & 0.58132929 & 0.49193138 & 0.536630335 \\
\hline Fczy & 0.678039919 & 0.651980945 & 0.665010432 \\
\hline Fayy & 0.64776847 & 0.593446924 & 0.620607697 \\
\hline Fosun Pharm & 0.469682442 & 0.744647296 & 0.607164869 \\
\hline Fuxiang & 0.699215567 & 0.600348021 & 0.649781794 \\
\hline Guangji & 0.686416693 & 0.640838606 & 0.66362765 \\
\hline Kwong Sang Hong & 0.739991067 & 0.650835677 & 0.695413372 \\
\hline Guang Yuyuan & 0.697803931 & 0.643573172 & 0.670688552 \\
\hline Lark & 0.630381464 & 0.657746723 & 0.644064093 \\
\hline Glsj & 0.677120987 & 0.682796241 & 0.679958614 \\
\hline Sinopharm Hyundai & 0.496221436 & 0.397513751 & 0.446867593 \\
\hline Harbin Pharm & 0.34428928 & 0.475791143 & 0.410040211 \\
\hline Haili Bio & 0.727883095 & 0.652682123 & 0.690282609 \\
\hline Hnhy & 0.609983775 & 0.528580996 & 0.569282386 \\
\hline Hepalink & 0.64389566 & 0.594403859 & 0.619149759 \\
\hline Haishun New Pharma & 0.750928424 & 0.661675715 & 0.706302069 \\
\hline Haisco & 0.720451696 & 0.487743987 & 0.604097841 \\
\hline Hisoar & 0.645544623 & 0.65460421 & 0.650074417 \\
\hline Haixin & 0.627599087 & 0.672097746 & 0.649848417 \\
\hline Hisun & 0.353460494 & 0.409015554 & 0.381238024 \\
\hline Han Sen Pharm & 0.668777752 & 0.663899646 & 0.666338699 \\
\hline Hybio & 0.636033086 & 0.580810237 & 0.608421662 \\
\hline Hengrui & 0.837490527 & 0.961302739 & 0.899396633 \\
\hline Chase Sun & 0.547733919 & 0.410016 & 0.478874959 \\
\hline NCPC & 0.36259657 & 0.447801928 & 0.405199249 \\
\hline Huahai & 0.543995409 & 0.553533665 & 0.548764537 \\
\hline Hualan Bio & 0.807931213 & 0.595846914 & 0.701889064 \\
\hline Huaren & 0.595328472 & 0.659644838 & 0.627486655 \\
\hline HRSJ & 0.541774861 & 0.574425738 & 0.558100299 \\
\hline China Resources Double-Crane & 0.568038828 & 0.54622275 & 0.557130789 \\
\hline Huashen Technology & 0.719199418 & 0.674035569 & 0.696617493 \\
\hline Walter Dyne & 0.664596536 & 0.687196219 & 0.675896377 \\
\hline Yanbian FC & 0.778142258 & 0.63590963 & 0.707025944 \\
\hline Kyrgyzstan & 0.601616926 & 0.496027976 & 0.548822451 \\
\hline Jichuan & 0.683807204 & 0.487854907 & 0.585831056 \\
\hline Jimin & 0.681185309 & 0.657322504 & 0.669253906 \\
\hline JYPC & 0.685562921 & 0.649641211 & 0.667602066 \\
\hline Joincare pharm & 0.610082457 & 0.454142296 & 0.532112376 \\
\hline Jiangzhong & 0.642567847 & 0.736567664 & 0.689567756 \\
\hline Jincheng & 0.639785389 & 0.569178368 & 0.604481878 \\
\hline
\end{tabular}


TABle 2: Continued.

\begin{tabular}{|c|c|c|c|}
\hline Company name & The first stage & The second stage & Total \\
\hline Jinhe Bio & 0.623354577 & 0.649028661 & 0.636191619 \\
\hline Jinling & 0.546235719 & 0.635576844 & 0.590906281 \\
\hline Jinshiya & 0.715796667 & 0.644757882 & 0.680277274 \\
\hline Jingxin & 0.68571291 & 0.570388142 & 0.628050526 \\
\hline Jinghua & 0.636944978 & 0.641649287 & 0.639297132 \\
\hline Jingfeng & 0.606488959 & 0.543530495 & 0.575009727 \\
\hline Jiuqiang & 0.786956228 & 0.700046207 & 0.743501218 \\
\hline Jiuzhitang & 0.645201026 & 0.516603024 & 0.580902025 \\
\hline Jiuzhou & 0.628567115 & 0.584155422 & 0.606361268 \\
\hline CONBA & 0.543861375 & 0.481004348 & 0.512432862 \\
\hline Kanghong & 0.665245011 & 0.527460836 & 0.596352924 \\
\hline Kangyuan & 0.61414204 & 0.577926992 & 0.596034516 \\
\hline Kangzhi & 0.684816087 & 0.656415871 & 0.670615979 \\
\hline $\mathrm{KHB}$ & 0.727976507 & 0.591841298 & 0.659908903 \\
\hline Kelun & 0.378799441 & 0.493231698 & 0.43601557 \\
\hline Sunflower & 0.556170097 & 0.511400244 & 0.533785171 \\
\hline Kunming Pharm & 0.534324416 & 0.551964191 & 0.543144303 \\
\hline Lummy & 0.625056228 & 0.628112755 & 0.626584491 \\
\hline LAYN & 0.721614484 & 0.661566479 & 0.691590481 \\
\hline Lisheng Pharma & 0.657353855 & 0.679177139 & 0.668265497 \\
\hline Livzon Pharm & 0.71738134 & 0.628871094 & 0.673126217 \\
\hline LEADMAN & 0.714944289 & 0.624498345 & 0.669721317 \\
\hline Lianhuan pharm & 0.698888411 & 0.670367118 & 0.684627765 \\
\hline Lingkang & 0.725743874 & 0.584694518 & 0.655219196 \\
\hline Lingrui Pharm & 0.666997747 & 0.677070779 & 0.672034263 \\
\hline Long jin Pharm & 0.753570272 & 0.647133166 & 0.700351719 \\
\hline Lukang Pharm & 0.509027638 & 0.460965245 & 0.484996442 \\
\hline Mike Bio & 0.742422513 & 0.650407694 & 0.696415103 \\
\hline M.k. & 0.654220437 & 0.562356283 & 0.60828836 \\
\hline Palin Bio & 0.703269836 & 0.65109186 & 0.677180848 \\
\hline PIEN TZE HUANG & 0.763824558 & 0.726005071 & 0.744914814 \\
\hline Julie Plec & 0.709624166 & 0.469789228 & 0.589706697 \\
\hline plyy & 0.489613809 & 0.53054052 & 0.510077164 \\
\hline CHEEZHENGTTM & 0.714689814 & 0.613582646 & 0.66413623 \\
\hline Qidi & 0.691053939 & 0.654977334 & 0.673015636 \\
\hline Qianhong Biopharma & 0.70228214 & 0.679203457 & 0.690742799 \\
\hline Qianjin Pharm & 0.612647181 & 0.607590824 & 0.610119003 \\
\hline Qianyuan & 0.664973414 & 0.556845497 & 0.610909455 \\
\hline Renfu & 0.322406425 & 0.42016555 & 0.371285988 \\
\hline Renhe Pharmacy & 0.596351472 & 0.672679619 & 0.634515546 \\
\hline rpsw & 0.651166925 & 0.5912946 & 0.621230762 \\
\hline Saisheng & 0.761947778 & 0.701836844 & 0.731892311 \\
\hline SAM & 0.671926336 & 0.659700513 & 0.665813424 \\
\hline Shanhe Pharmacy & 0.734791349 & 0.650221186 & 0.692506268 \\
\hline Shkb & 0.698069521 & 0.680387876 & 0.689228698 \\
\hline Shanghai RAAS Blood Products & 0.676407378 & 0.605561928 & 0.640984653 \\
\hline Shenqi & 0.646589105 & 0.581969742 & 0.614279423 \\
\hline Biological Stock & 0.760202981 & 0.756042924 & 0.758122952 \\
\hline Salvage Pharm & 0.463144872 & 0.502413614 & 0.482779243 \\
\hline Sts & 0.739781567 & 0.689149244 & 0.714465406 \\
\hline Scyy & 0.699966811 & 0.603380282 & 0.651673546 \\
\hline Beijing SL Pharm & 0.773760654 & 0.68883442 & 0.731297537 \\
\hline Stellite & 0.667760149 & 0.642702048 & 0.655231099 \\
\hline Shsw & 0.73692057 & 0.657203864 & 0.697062217 \\
\hline Tat & 0.60265023 & 0.544697259 & 0.573673745 \\
\hline Taiji Group & 0.399279671 & 0.498518625 & 0.448899148 \\
\hline Taloph Pharm & 0.61924255 & 0.644434623 & 0.631838587 \\
\hline Teyi & 0.698667383 & 0.66716684 & 0.682917112 \\
\hline Tasly & 0.41666853 & 0.630275487 & 0.523472009 \\
\hline Tiantan Biological & 0.733010668 & 0.657600328 & 0.695305498 \\
\hline
\end{tabular}


TABLE 2: Continued.

\begin{tabular}{|c|c|c|c|}
\hline Company name & The first stage & The second stage & Total \\
\hline Tianyao Pharm & 0.646240088 & 0.613143357 & 0.629691722 \\
\hline Thdb & 0.77883326 & 0.706539764 & 0.742686512 \\
\hline Thjm & 0.567809163 & 0.564386742 & 0.566097953 \\
\hline TRT & 0.499628521 & 0.691621404 & 0.595624963 \\
\hline Wanbangde & 0.427406852 & 0.537992679 & 0.482699766 \\
\hline Wondfo & 0.715009555 & 0.631798868 & 0.673404211 \\
\hline WEDGE INDUSTRIAL & 0.736184308 & 0.643355439 & 0.689769873 \\
\hline Weiming & 0.711826016 & 0.650321829 & 0.681073922 \\
\hline Wowu & 0.770567021 & 0.675840362 & 0.723203692 \\
\hline Wohua & 0.703903392 & 0.661188132 & 0.682545762 \\
\hline Wosen & 0.671912477 & 0.552904143 & 0.61240831 \\
\hline AMD & 0.750945781 & 0.61052309 & 0.680734436 \\
\hline Xianju Pharm & 0.609803878 & 0.607517262 & 0.60866057 \\
\hline Xiangxue Pharm & 0.56837251 & 0.537740093 & 0.553056301 \\
\hline Sunflower & 0.55841059 & 0.566683692 & 0.562547141 \\
\hline NHU & 0.632654979 & 0.4122508 & 0.52245289 \\
\hline Xinhua & 0.488947377 & 0.556767052 & 0.522857214 \\
\hline Xinbang & 0.440203247 & 0.395079286 & 0.417641267 \\
\hline SALUBRIS & 0.715240078 & 0.63026292 & 0.672751499 \\
\hline BROTHER & 0.697594871 & 0.621553604 & 0.659574237 \\
\hline Yabao & 0.558060463 & 0.567098443 & 0.562579453 \\
\hline Yatai & 0.59468608 & 0.568673089 & 0.581679584 \\
\hline Yananbikang & 0.585080092 & 0.503340182 & 0.544210137 \\
\hline Yiling Pharm & 0.591814993 & 0.635212897 & 0.613513945 \\
\hline Yifan & 0.659880029 & 0.609050879 & 0.634465454 \\
\hline Yibai & 0.564093323 & 0.437330699 & 0.500712011 \\
\hline Yisheng & 0.661646927 & 0.603737031 & 0.632691979 \\
\hline Yiduoli & 0.648099384 & 0.597623845 & 0.622861614 \\
\hline Chinataurine & 0.692881108 & 0.647370414 & 0.670125761 \\
\hline Gloria Pharm & 0.479739963 & 0.433218753 & 0.456479358 \\
\hline Baiyao & 0.50362558 & 0.819177469 & 0.661401524 \\
\hline Zhejiang Medicine & 0.500873109 & 0.515343568 & 0.508108338 \\
\hline Zhenbao Island & 0.66468048 & 0.494783002 & 0.579731741 \\
\hline zdzy & 0.568909321 & 0.561712443 & 0.565310882 \\
\hline Zhifei & 0.821043844 & 0.783585599 & 0.802314721 \\
\hline Zhongguancun & 0.603226518 & 0.546917339 & 0.575071929 \\
\hline China Medicine & 0.326022175 & 0.619902235 & 0.472962205 \\
\hline Zhongheng Group & 0.699336493 & 0.716431982 & 0.707884238 \\
\hline Zhongmu & 0.534061049 & 0.625219552 & 0.579640301 \\
\hline Zhongxin & 0.523656226 & 0.64705675 & 0.585356488 \\
\hline Zsyy & 0.728308394 & 0.611906374 & 0.670107384 \\
\hline JLZX & 0.70032643 & 0.554290101 & 0.627308266 \\
\hline Zuoli & 0.658487779 & 0.492143686 & 0.575315732 \\
\hline
\end{tabular}

Biological is below 0.6 in the two stages, for the resources cannot be well utilized (see Table 4 for specific annual data).

4.5. Overall Efficiency Analysis. In the overall efficiency analysis (shown in Table 2), there are 109 companies with efficiency between 0.6 and 1.0, accounting for $66.9 \%$, and it shows that the technical efficiency of the second stage is less than that of the first stage.

Comparing Hengrui (the highest efficiency) and Baiyunshan (the lowest efficiency), we found that Hengrui did not receive government subsidies in the 2018 and 2019, but the technical efficiency reached 1, and Baiyunshan has received government subsidies, but the technical efficiency rises first and then decreases.
For Zhifei Bio with the second highest efficiency, its efficiency in 2018 and 2019 was significantly higher than in 2016 and 2017, and the government subsidies received by Zhifei Bio in 2018 and 2019 were significantly lower than before. The second-to-last-ranked company, Medicare, reached a low point in 2018, followed by a significant rebound next year, when it was not subsidized by the government in 2019.

It can be concluded that government subsidies have no obvious effect for most companies, but it has a positive impact on enterprises with low efficiency in a short term. The government may need to reconsider the way of subsidies to pharmaceutical companies, such as the capital subsidies to equipment upgrades and talent introduction (see Table 5 for specific annual data). 
TABLE 3: Analysis of DEA efficiency in the first stage of Chinese pharmaceutical companies.

\begin{tabular}{|c|c|c|c|c|}
\hline \multirow{2}{*}{ Company name } & \multicolumn{4}{|c|}{ Year } \\
\hline & $2015-2016$ & $2016-2017$ & $2017-2018$ & 2018-2019 \\
\hline Adisseo & 1 & 0.538826377 & 0.748769622 & 0.639633789 \\
\hline Anke Bio & 0.69893892 & 0.731620495 & 0.671113197 & 0.703361568 \\
\hline Osaikon & 0.713077361 & 0.75673785 & 0.813339088 & 0.817531461 \\
\hline Baiyunshan & 0.328542111 & 0.356320523 & 0.546507045 & 0.303672764 \\
\hline Bdyy & 0.589761232 & 0.62883886 & 0.592320492 & 0.673332429 \\
\hline Beilu & 0.701152973 & 0.769964623 & 0.718534493 & 0.832174827 \\
\hline Porton & 0.617509415 & 0.622015319 & 0.606350945 & 0.659897114 \\
\hline None & 0.709527587 & 0.749839674 & 0.680641517 & 0.692813179 \\
\hline Changchun High-Tech & 0.644238901 & 0.693875856 & 0.678445711 & 1 \\
\hline Changjiang Runfa Medicine & 0.661073921 & 0.633858532 & 0.577628241 & 0.617216502 \\
\hline Changshan Pharma & 0.702574435 & 0.737444456 & 0.654896914 & 0.726604025 \\
\hline DAJY & 0.608034181 & 0.612562673 & 0.565649446 & 0.659420187 \\
\hline Dezhan & 0.778248014 & 0.8859713 & 0.801172953 & 0.735162569 \\
\hline Jiao & 0.695876014 & 0.752555196 & 0.784627533 & 0.519464099 \\
\hline DBBT & 0.698427402 & 0.732910456 & 0.683799871 & 0.747814366 \\
\hline $\mathrm{VC}$ & 0.356746951 & 0.369605175 & 0.596620054 & 0.529513216 \\
\hline Dongcheng & 0.685298529 & 0.713356357 & 0.65224054 & 0.644469293 \\
\hline Nhwa Pharm & 0.566050744 & 0.595888879 & 0.559629612 & 0.90136936 \\
\hline Ekzy & 0.695893135 & 0.642142688 & 0.56111062 & 0.817019263 \\
\hline Fangsheng & 0.690430635 & 0.7072366 & 0.668234842 & 0.734875807 \\
\hline Fengyuan & 0.507954055 & 0.513382048 & 0.500312529 & 0.803668528 \\
\hline Fczy & 0.673318722 & 0.706559734 & 0.637287549 & 0.694993671 \\
\hline Fayy & 0.640199162 & 0.622882581 & 0.47487985 & 0.853112289 \\
\hline Fosun Pharm & 0.440829161 & 0.453177832 & 0.491647136 & 0.49307564 \\
\hline Fuxiang & 0.69509172 & 0.713319861 & 0.655317677 & 0.733133012 \\
\hline Guangji & 0.665875845 & 0.685465131 & 0.666509377 & 0.727816419 \\
\hline Kwong Sang Hong & 0.731211949 & 0.758118876 & 0.703598927 & 0.767034517 \\
\hline Guang Yuyuan & 0.705727447 & 0.728802756 & 0.672683165 & 0.684002357 \\
\hline Lark & 0.592069879 & 0.607690808 & 0.548144601 & 0.77362057 \\
\hline Glsj & 0.646076312 & 0.70291605 & 0.642642634 & 0.716848951 \\
\hline Sinopharm Hyundai & 0.550500314 & 0.31927442 & 0.586702033 & 0.528408978 \\
\hline Harbin Pharm & 0.232904004 & 0.239750504 & 0.452243584 & 0.452259029 \\
\hline Haili Bio & 0.720958825 & 0.755724092 & 0.683383826 & 0.751465636 \\
\hline Hnhy & 0.593723249 & 0.615663681 & 0.533902785 & 0.696645384 \\
\hline Hepalink & 0.613624689 & 0.594913319 & 0.565038631 & 0.802005999 \\
\hline Haishun New Pharma & 0.730858479 & 0.770599806 & 0.71368573 & 0.788569679 \\
\hline Haisco & 0.694950719 & 0.689640795 & 0.627724818 & 0.86949045 \\
\hline Hisoar & 0.536160514 & 0.598345145 & 0.58053481 & 0.867138023 \\
\hline Haixin & 0.598921852 & 0.651676624 & 0.60413451 & 0.655663364 \\
\hline Hisun & 0.279462381 & 0.291252982 & 0.396970805 & 0.446155807 \\
\hline Han Sen Pharm & 0.622641145 & 0.670338249 & 0.638264052 & 0.743867561 \\
\hline Hybio & 0.706990021 & 0.762095736 & 0.569815841 & 0.505230747 \\
\hline Hengrui & 0.659908876 & 0.690053233 & 1 & 1 \\
\hline Chase Sun & 0.590616729 & 0.546391865 & 0.485056058 & 0.568871023 \\
\hline NCPC & 0.261478601 & 0.273294764 & 0.476531644 & 0.439081271 \\
\hline Huahai & 0.510939772 & 0.532978902 & 0.422450272 & 0.70961269 \\
\hline Hualan Bio & 0.724489053 & 0.75903868 & 0.74819712 & 1 \\
\hline Huaren & 0.547811923 & 0.60655341 & 0.573018429 & 0.653930126 \\
\hline HRSJ & 0.421806748 & 0.424780128 & 0.642346121 & 0.678166446 \\
\hline China Resources Double-Crane & 0.396338204 & 0.412017952 & 0.746295359 & 0.717503796 \\
\hline Huashen Technology & 0.692282897 & 0.748644224 & 0.673121394 & 0.762749156 \\
\hline Walter Dyne & 0.64905781 & 0.687320156 & 0.604331968 & 0.717676209 \\
\hline Yanbian FC & 0.728434735 & 0.796358227 & 0.622973596 & 0.964802474 \\
\hline Kyrgyzstan & 0.690648563 & 0.723721435 & 0.676558269 & 0.315539437 \\
\hline Jichuan & 0.654593403 & 0.687752464 & 0.658917181 & 0.733965769 \\
\hline Jimin & 0.667207312 & 0.715203145 & 0.639062034 & 0.703268743 \\
\hline JYPC & 0.676163429 & 0.660551878 & 0.674564338 & 0.730972038 \\
\hline Joincare Pharm & 0.42609937 & 0.779556709 & 0.42013106 & 0.814542688 \\
\hline Jiangzhong & 0.577983092 & 0.660234497 & 0.621771461 & 0.710282338 \\
\hline
\end{tabular}


TABle 3: Continued.

\begin{tabular}{|c|c|c|c|c|}
\hline \multirow{2}{*}{ Company name } & \multicolumn{4}{|c|}{ Year } \\
\hline & $2015-2016$ & $2016-2017$ & $2017-2018$ & 2018-2019 \\
\hline Jincheng & 0.604349975 & 0.635628024 & 0.540328079 & 0.778835478 \\
\hline Jinhe Bio & 0.620488457 & 0.622315827 & 0.593163862 & 0.657450163 \\
\hline Jinling & 0.457693647 & 0.464485434 & 0.485300003 & 0.77746379 \\
\hline Jinshiya & 0.739579461 & 0.794884842 & 0.642073972 & 0.686648392 \\
\hline Jingxin & 0.615013047 & 0.645371162 & 0.600849303 & 0.881618128 \\
\hline Jinghua & 0.654292046 & 0.695723085 & 0.644925134 & 0.552839647 \\
\hline Jingfeng & 0.655470879 & 0.625155781 & 0.56168705 & 0.583642128 \\
\hline Jiuqiang & 0.775537026 & 0.80576769 & 0.741353597 & 0.825166597 \\
\hline Jiuzhitang & 0.638432251 & 0.632345693 & 0.526816311 & 0.783209851 \\
\hline Jiuzhou & 0.531129929 & 0.563082683 & 0.553227164 & 0.866828683 \\
\hline CONBA & 0.424959572 & 0.448091924 & 0.76318362 & 0.539210385 \\
\hline Kanghong & 0.649678902 & 0.676913482 & 0.617801313 & 0.716586348 \\
\hline Kangyuan & 0.547173659 & 0.538412405 & 0.521974436 & 0.84900766 \\
\hline Kangzhi & 0.681459875 & 0.718856205 & 0.658583409 & 0.68036486 \\
\hline KHB & 0.679909201 & 0.70601526 & 0.646219781 & 0.879761787 \\
\hline Kelun & 0.309440068 & 0.306632161 & 0.481370093 & 0.417755441 \\
\hline Sunflower & 0.503468625 & 0.542712714 & 0.541164765 & 0.637334286 \\
\hline Kunming Pharm & 0.487752766 & 0.494592469 & 0.456784207 & 0.698168222 \\
\hline Lummy & 0.59477378 & 0.650148325 & 0.620139476 & 0.63516333 \\
\hline LAYN & 0.706666281 & 0.764250345 & 0.658484063 & 0.757057246 \\
\hline Lisheng Pharma & 0.652358213 & 0.658225585 & 0.633802648 & 0.685028972 \\
\hline Livzon Pharm & 0.483127888 & 0.90147395 & 0.765854345 & 0.719069177 \\
\hline LEADMAN & 0.685277868 & 0.739693609 & 0.685479669 & 0.749326011 \\
\hline Lianhuan Pharm & 0.672599294 & 0.711757269 & 0.670159541 & 0.741037539 \\
\hline Lingkang & 0.698902905 & 0.743402231 & 0.696586279 & 0.764084079 \\
\hline Lingrui Pharm & 0.674401636 & 0.672389024 & 0.614906134 & 0.706294196 \\
\hline Long Jin Pharm & 0.731845112 & 0.770452444 & 0.718767022 & 0.793216512 \\
\hline Lukang Pharm & 0.427468486 & 0.46570682 & 0.460332769 & 0.682602477 \\
\hline Mike Bio & 0.708027483 & 0.72319852 & 0.645149742 & 0.893314305 \\
\hline M.k. & 0.708395924 & 0.726482926 & 0.56873759 & 0.613265307 \\
\hline Palin Bio & 0.671391314 & 0.718181986 & 0.666425242 & 0.757080803 \\
\hline PIEN TZE HUANG & 0.684930611 & 0.750405659 & 0.661039196 & 0.958922766 \\
\hline Julie Plec & 0.707704329 & 0.723714045 & 0.673462726 & 0.733615566 \\
\hline Plyy & 0.406959748 & 0.414853778 & 0.427735839 & 0.708905869 \\
\hline CHEEZHENGTTM & 0.686682951 & 0.725135357 & 0.678237865 & 0.768703082 \\
\hline Qidi & 0.666806547 & 0.68236924 & 0.64498398 & 0.770055989 \\
\hline Qianhong Biopharma & 0.71938682 & 0.719385025 & 0.656560098 & 0.713796618 \\
\hline Qianjin Pharm & 0.530656791 & 0.548629069 & 0.522713754 & 0.848589111 \\
\hline Qianyuan & 0.638506467 & 0.671543368 & 0.62791026 & 0.721933561 \\
\hline Renfu & 0.334218803 & 0.413272744 & 0.149231487 & 0.392902669 \\
\hline Renhe Pharmacy & 0.533268479 & 0.506914813 & 0.519999497 & 0.8252231 \\
\hline Rpsw & 0.638039028 & 0.654974398 & 0.617845497 & 0.693808777 \\
\hline Saisheng & 0.754473392 & 0.796748489 & 0.72770648 & 0.76886275 \\
\hline SAM & 0.680909431 & 0.689598358 & 0.594020614 & 0.72317694 \\
\hline Shanhe Pharmacy & 0.713262981 & 0.747755843 & 0.701291618 & 0.776854953 \\
\hline Shkb & 0.690613654 & 0.721026937 & 0.655361928 & 0.725275566 \\
\hline Shanghai RAAS Blood Products & 0.815937216 & 0.711632966 & 0.29512233 & 0.882937 \\
\hline Shenqi & 0.646515892 & 0.665122901 & 0.604905717 & 0.669811909 \\
\hline Biological Stock & 0.776300364 & 0.857731961 & 0.733310511 & 0.673469086 \\
\hline Salvage Pharm & 0.405423981 & 0.471147411 & 0.570580408 & 0.405427689 \\
\hline Sts & 0.745342868 & 0.789809576 & 0.695943443 & 0.728030381 \\
\hline Scyy & 0.621324992 & 0.736624764 & 0.67014276 & 0.771774728 \\
\hline Beijing SL Pharm & 0.754959569 & 0.822479251 & 0.739066637 & 0.778537157 \\
\hline Stellite & 0.64699861 & 0.679791002 & 0.648708242 & 0.695542742 \\
\hline Shsw & 0.706490688 & 0.749047972 & 0.701812101 & 0.790331518 \\
\hline Tat & 0.583302035 & 0.579802833 & 0.530612746 & 0.716883304 \\
\hline Taiji Group & 0.368697953 & 0.288373447 & 0.505506669 & 0.434540617 \\
\hline Taloph Pharm & 0.60505421 & 0.644707882 & 0.569453781 & 0.657754327 \\
\hline Teyi & 0.685639388 & 0.710137044 & 0.667309716 & 0.731583384 \\
\hline
\end{tabular}


TABle 3: Continued.

\begin{tabular}{|c|c|c|c|c|}
\hline \multirow{2}{*}{ Company name } & \multicolumn{4}{|c|}{ Year } \\
\hline & 2015-2016 & 2016-2017 & 2017-2018 & 2018-2019 \\
\hline Tasly & 0.344736323 & 0.364398246 & 0.547507961 & 0.410031592 \\
\hline Tiantan Biological & 0.56685493 & 0.735139685 & 0.705597237 & 0.924450822 \\
\hline Tianyao Pharm & 0.567784075 & 0.640100799 & 0.568366328 & 0.808709151 \\
\hline Thdb & 0.690420152 & 0.76073724 & 0.692058634 & 0.972117015 \\
\hline Thjm & 0.700759308 & 0.682066065 & 0.62099078 & 0.2674205 \\
\hline TRT & 0.449941637 & 0.452561856 & 0.578565313 & 0.517445278 \\
\hline Wanbangde & 0.350398153 & 0.410898312 & 0.464547495 & 0.483783447 \\
\hline Wondfo & 0.706033933 & 0.749163259 & 0.666377487 & 0.738463539 \\
\hline WEDGE INDUSTRIAL & 0.718041181 & 0.768397698 & 0.690260111 & 0.76803824 \\
\hline Weiming & 0.748177009 & 0.769128321 & 0.619853486 & 0.710145248 \\
\hline Wowu & 0.739298451 & 0.784243922 & 0.7386125 & 0.820113211 \\
\hline Wohua & 0.674322938 & 0.710157045 & 0.659012071 & 0.772121516 \\
\hline Wosen & 0.619303412 & 0.571491875 & 0.797393821 & 0.699460802 \\
\hline AMD & 0.689966569 & 0.789941263 & 0.71329527 & 0.810580022 \\
\hline Xianju Pharm & 0.514464857 & 0.566490622 & 0.540109586 & 0.818150446 \\
\hline Xiangxue Pharm & 0.537783667 & 0.540068623 & 0.481275233 & 0.714362519 \\
\hline Sunflower & 0.540029523 & 0.598998632 & 0.388532054 & 0.706082152 \\
\hline NHU & 0.517692055 & 0.603883517 & 0.66053792 & 0.748506424 \\
\hline Xinhua & 0.402109863 & 0.430156322 & 0.437174929 & 0.686348395 \\
\hline Xinbang & 0.43011624 & 0.428808192 & 0.361237255 & 0.540651302 \\
\hline SALUBRIS & 0.744881615 & 0.779294136 & 0.697651488 & 0.639133074 \\
\hline BROTHER & 0.663383853 & 0.719528977 & 0.583598178 & 0.823868476 \\
\hline Yabao & 0.453418706 & 0.507914392 & 0.515088977 & 0.755819777 \\
\hline Yatai & 0.687624616 & 0.723497946 & 0.658661044 & 0.308960713 \\
\hline Yananbikang & 0.615088274 & 0.598667978 & 0.63509745 & 0.491466664 \\
\hline Yiling Pharm & 0.535399897 & 0.54629301 & 0.521004694 & 0.764562368 \\
\hline Yifan & 0.62137248 & 0.706145232 & 0.543427556 & 0.768574846 \\
\hline Yibai & 0.601819983 & 0.583245952 & 0.344474902 & 0.726832453 \\
\hline Yisheng & 0.640708057 & 0.679203014 & 0.623539381 & 0.703137255 \\
\hline Yiduoli & 0.61878647 & 0.601097854 & 0.566968291 & 0.80554492 \\
\hline Chinataurine & 0.65392248 & 0.715865276 & 0.674513572 & 0.727223103 \\
\hline Gloria Pharm & 0.619802462 & 0.583161465 & 0.534749776 & 0.181246147 \\
\hline Baiyao & 0.407703292 & 0.415325832 & 0.598683278 & 0.592789916 \\
\hline Zhejiang Medicine & 0.39694149 & 0.393998731 & 0.627437898 & 0.585114319 \\
\hline Zhenbao Island & 0.615637011 & 0.630467145 & 0.565464187 & 0.847153576 \\
\hline Zdzy & 0.53930568 & 0.541502435 & 0.447281659 & 0.74754751 \\
\hline Zhifei & 0.659011263 & 0.779383924 & 0.84578019 & 1 \\
\hline Zhongguancun & 0.607984942 & 0.597661057 & 0.559189938 & 0.648070137 \\
\hline China Medicine & 0.298293719 & 0.284321785 & 0.389396559 & 0.332076638 \\
\hline Zhongheng Group & 0.6583369 & 0.730823738 & 0.660884453 & 0.747300882 \\
\hline Zhongmu & 0.46697542 & 0.505701728 & 0.480753041 & 0.682814008 \\
\hline Zhongxin & 0.425869997 & 0.478604566 & 0.475336345 & 0.714813998 \\
\hline Zsyy & 0.672284742 & 0.721839471 & 0.64125814 & 0.877851223 \\
\hline JLZX & 0.658045275 & 0.724715509 & 0.596666189 & 0.821878746 \\
\hline Zuoli & 0.647050689 & 0.667106722 & 0.616372459 & 0.703421246 \\
\hline
\end{tabular}


TABLE 4: Analysis of DEA efficiency in the second stage of Chinese pharmaceutical companies.

\begin{tabular}{|c|c|c|c|c|}
\hline \multirow{2}{*}{ Company name } & \multicolumn{4}{|c|}{ Year } \\
\hline & 2015-2016 & 2016-2017 & 2017-2018 & 2018-2019 \\
\hline Adisseo & 1 & 0.649837545 & 0.625737431 & 0.541530825 \\
\hline Anke Bio & 0.765162026 & 0.476189062 & 0.772255149 & 0.613658522 \\
\hline Osaikon & 0.843778066 & 0.441445985 & 0.850780731 & 0.575021161 \\
\hline Baiyunshan & 0.128029731 & 0.338472809 & 0.44681541 & 0.404956323 \\
\hline Bdyy & 0.890834395 & 0.445222754 & 0.727676509 & 0.608956969 \\
\hline Beilu & 0.808544991 & 0.447781474 & 0.735202266 & 0.658558851 \\
\hline Porton & 0.790606856 & 0.452864356 & 0.741151893 & 0.630953461 \\
\hline None & 0.899553352 & 0.412224769 & 0.692164738 & 0.575225616 \\
\hline Changchun High-Tech & 0.697331634 & 0.534086802 & 0.974210731 & 0.83805388 \\
\hline Changjiang Runfa Medicine & 0.758278202 & 0.457208597 & 0.757178228 & 0.355161748 \\
\hline Changshan Pharma & 0.839430913 & 0.452268392 & 0.722949426 & 0.63821128 \\
\hline DAJY & 0.498749812 & 0.453046771 & 0.736062102 & 0.592503277 \\
\hline Dezhan & 1 & 0.540144516 & 0.907325608 & 0.65741689 \\
\hline Jiao & 1 & 0.695657355 & 1 & 0.349390869 \\
\hline $\mathrm{DBBT}$ & 0.79160979 & 0.443887279 & 0.725436161 & 0.605439407 \\
\hline $\mathrm{VC}$ & 0.52176685 & 0.452560475 & 0.4923262 & 0.401474275 \\
\hline Dongcheng & 0.875632334 & 0.456954862 & 0.768638857 & 0.621583435 \\
\hline Nhwa Pharm & 0.902685534 & 0.481101741 & 0.812506347 & 0.47310525 \\
\hline Ekzy & 0.663965694 & 0.492139653 & 0.741589175 & 0.417137904 \\
\hline Fangsheng & 0.83728348 & 0.445912668 & 0.729448377 & 0.613888946 \\
\hline Fengyuan & 0.381700772 & 0.448248178 & 0.729255255 & 0.408521314 \\
\hline Fczy & 0.810829455 & 0.450323096 & 0.733612414 & 0.613158813 \\
\hline Fayy & 0.853851088 & 0.462652138 & 0.626676033 & 0.430608438 \\
\hline Fosun Pharm & 0.405879589 & 0.887608474 & 0.863039604 & 0.822061518 \\
\hline Fuxiang & 0.686147937 & 0.40302621 & 0.65968793 & 0.652530008 \\
\hline Guangji & 0.781783744 & 0.438862422 & 0.728105584 & 0.614602675 \\
\hline Kwong Sang Hong & 0.833600898 & 0.445235421 & 0.721873605 & 0.602632782 \\
\hline Guang Yuyuan & 0.873544633 & 0.467173716 & 0.78987118 & 0.443703159 \\
\hline Lark & 0.856359723 & 0.507355275 & 0.834541643 & 0.432730249 \\
\hline Glsj & 0.762864498 & 0.498932775 & 0.802401553 & 0.666986138 \\
\hline Sinopharm Hyundai & 0.304326441 & 0.467872765 & 0.522609903 & 0.295245894 \\
\hline Harbin Pharm & 0.471907832 & 0.498927974 & 0.51892983 & 0.413398936 \\
\hline Haili Bio & 0.845981594 & 0.452963586 & 0.715761969 & 0.596021344 \\
\hline Hnhy & 0.546132286 & 0.451209071 & 0.73929906 & 0.377683569 \\
\hline Hepalink & 0.570692829 & 0.455773586 & 0.833748227 & 0.517400795 \\
\hline Haishun New Pharma & 0.875191121 & 0.441283929 & 0.718583719 & 0.61164409 \\
\hline Haisco & 0.265247759 & 0.462094574 & 0.770404638 & 0.453228976 \\
\hline Hisoar & 0.806777693 & 0.483733043 & 0.841284576 & 0.486621529 \\
\hline Haixin & 0.869599312 & 0.45122025 & 0.748838002 & 0.618733421 \\
\hline Hisun & 0.295330677 & 0.469935051 & 0.434672128 & 0.436124359 \\
\hline Han Sen Pharm & 0.842792274 & 0.449054927 & 0.739269782 & 0.624481603 \\
\hline Hybio & 0.741190863 & 0.482159427 & 0.64940653 & 0.450484128 \\
\hline Hengrui & 0.994006619 & 0.851204335 & 1 & 1 \\
\hline Chase Sun & 0.510934768 & 0.252963632 & 0.388183145 & 0.487982456 \\
\hline NCPC & 0.446958138 & 0.442893623 & 0.485019512 & 0.416336438 \\
\hline Huahai & 0.483631419 & 0.517819876 & 0.744562547 & 0.468120819 \\
\hline Hualan Bio & 0.488018688 & 0.463275133 & 0.825155867 & 0.606937968 \\
\hline Huaren & 0.858322442 & 0.445957989 & 0.72614299 & 0.608155932 \\
\hline HRSJ & 0.39055996 & 0.606296731 & 0.659827456 & 0.641018804 \\
\hline China Resources Double-Crane & 0.532023886 & 0.544202986 & 0.588679129 & 0.519985 \\
\hline Huashen Technology & 0.899423723 & 0.461862909 & 0.723906959 & 0.610948685 \\
\hline Walter Dyne & 0.842502696 & 0.497242721 & 0.767143201 & 0.641896259 \\
\hline Yanbian FC & 0.442623386 & 0.657329408 & 0.887815963 & 0.555869763 \\
\hline Kyrgyzstan & 0.455180857 & 0.466836149 & 0.763056261 & 0.299038639 \\
\hline Jichuan & 0.692456078 & 0.269019483 & 0.480891191 & 0.509052877 \\
\hline Jimin & 0.843175982 & 0.447230193 & 0.72593401 & 0.612949828 \\
\hline JYPC & 0.902264504 & 0.405752965 & 0.710764503 & 0.579782874 \\
\hline Joincare Pharm & 0.320679344 & 0.446019187 & 0.442365325 & 0.607505328 \\
\hline Jiangzhong & 0.968578156 & 0.488910163 & 0.806995681 & 0.681786657 \\
\hline
\end{tabular}


TABle 4: Continued.

\begin{tabular}{|c|c|c|c|c|}
\hline \multirow{2}{*}{ Company name } & \multicolumn{4}{|c|}{ Year } \\
\hline & $2015-2016$ & 2016-2017 & 2017-2018 & 2018-2019 \\
\hline Jincheng & 0.680587626 & 0.446883725 & 0.726463433 & 0.422778688 \\
\hline Jinhe Bio & 0.767555971 & 0.454800853 & 0.751896726 & 0.621861095 \\
\hline Jinling & 0.882151804 & 0.462632532 & 0.777149818 & 0.420373223 \\
\hline Jinshiya & 0.818824975 & 0.430337738 & 0.708029409 & 0.621839405 \\
\hline Jingxin & 0.754271786 & 0.3997261 & 0.669011225 & 0.458543459 \\
\hline Jinghua & 0.818896414 & 0.46479897 & 0.771305173 & 0.511596589 \\
\hline Jingfeng & 0.659628837 & 0.462586912 & 0.756245434 & 0.295660795 \\
\hline Jiuqiang & 0.888800059 & 0.475114126 & 0.779541159 & 0.656729486 \\
\hline Jiuzhitang & 0.682939594 & 0.388385296 & 0.576221175 & 0.418866032 \\
\hline Jiuzhou & 0.70068303 & 0.459376494 & 0.75017596 & 0.426386203 \\
\hline CONBA & 0.450840367 & 0.532007534 & 0.572159801 & 0.36900969 \\
\hline Kanghong & 0.630861917 & 0.383874254 & 0.632910424 & 0.462196751 \\
\hline Kangyuan & 0.569889462 & 0.483812562 & 0.800075831 & 0.457930115 \\
\hline Kangzhi & 0.860448512 & 0.447077729 & 0.722807424 & 0.595329819 \\
\hline KHB & 0.75874045 & 0.446262253 & 0.731865553 & 0.430496937 \\
\hline Kelun & 0.392192554 & 0.491878068 & 0.573939315 & 0.514916857 \\
\hline Sunflower & 0.397069408 & 0.402221867 & 0.677747189 & 0.568562512 \\
\hline Kunming Pharm & 0.484712539 & 0.482852557 & 0.787863272 & 0.452428396 \\
\hline Lummy & 0.757591911 & 0.446024294 & 0.735699424 & 0.57313539 \\
\hline LAYN & 0.82616477 & 0.46673708 & 0.735088104 & 0.618275961 \\
\hline Lisheng Pharma & 0.892289898 & 0.44823865 & 0.743782606 & 0.632397402 \\
\hline Livzon Pharm & 0.330476519 & 1 & 0.621269109 & 0.563738749 \\
\hline LEADMAN & 0.744491132 & 0.439674197 & 0.708587278 & 0.605240773 \\
\hline Lianhuan Pharm & 0.883381664 & 0.449882686 & 0.733590338 & 0.614613785 \\
\hline Lingkang & 0.487342648 & 0.461147315 & 0.755609423 & 0.634678684 \\
\hline Lingrui Pharm & 0.820570294 & 0.469108287 & 0.768403276 & 0.650201258 \\
\hline Long Jin Pharm & 0.825269505 & 0.445474296 & 0.7213483 & 0.596440566 \\
\hline Lukang Pharm & 0.709307544 & 0.271967764 & 0.448405964 & 0.41417971 \\
\hline Mike Bio & 0.829672491 & 0.491963544 & 0.816516452 & 0.463478288 \\
\hline M.k. & 0.702540171 & 0.461798568 & 0.750504723 & 0.334581671 \\
\hline Palin Bio & 0.800366688 & 0.44299407 & 0.733746558 & 0.627260125 \\
\hline PIEN TZE HUANG & 0.9045343 & 0.538290845 & 0.947536174 & 0.513658965 \\
\hline Julie Plec & 0.549521085 & 0.305111815 & 0.499939327 & 0.524584684 \\
\hline Plyy & 0.533402894 & 0.421972728 & 0.708018213 & 0.458768245 \\
\hline CHEEZHENGTTM & 0.565148659 & 0.465429448 & 0.761471867 & 0.662280612 \\
\hline Qidi & 0.86117892 & 0.441527153 & 0.709508207 & 0.607695056 \\
\hline Qianhong Biopharma & 0.874606048 & 0.456315537 & 0.750563573 & 0.635328671 \\
\hline Qianjin Pharm & 0.739246108 & 0.471814504 & 0.779924867 & 0.439377818 \\
\hline Qianyuan & 0.831338985 & 0.404575277 & 0.655154668 & 0.336313058 \\
\hline Renfu & 0.334414205 & 0.618045999 & 0.176055483 & 0.552146513 \\
\hline Renhe Pharmacy & 0.888111873 & 0.496037076 & 0.837040815 & 0.469528713 \\
\hline Rpsw & 0.594394568 & 0.433880869 & 0.709930487 & 0.626972475 \\
\hline Saisheng & 0.929682934 & 0.476157778 & 0.77599003 & 0.625516632 \\
\hline SAM & 0.905264682 & 0.451150287 & 0.680555861 & 0.60183122 \\
\hline Shanhe Pharmacy & 0.847924844 & 0.432928146 & 0.710008765 & 0.610022991 \\
\hline Shkb & 0.848089711 & 0.471499157 & 0.758909287 & 0.643053348 \\
\hline Shanghai RAAS Blood Products & 1 & 0.54470828 & 0.409520354 & 0.468019077 \\
\hline Shenqi & 0.714762096 & 0.381344561 & 0.617613041 & 0.61415927 \\
\hline Biological Stock & 0.965359357 & 0.549223179 & 0.870620483 & 0.638968678 \\
\hline Salvage Pharm & 0.689364433 & 0.415308201 & 0.706749477 & 0.198232344 \\
\hline Sts & 0.935757517 & 0.472141333 & 0.74357052 & 0.605127606 \\
\hline Scyy & 0.739153533 & 0.426485993 & 0.676414771 & 0.571466831 \\
\hline Beijing SL Pharm & 0.733749939 & 0.506947781 & 0.832842848 & 0.681797114 \\
\hline Stellite & 0.803378896 & 0.437370993 & 0.715251262 & 0.614807042 \\
\hline Shsw & 0.890410616 & 0.434761769 & 0.700177597 & 0.603465476 \\
\hline Tat & 0.842586294 & 0.360807923 & 0.584823244 & 0.390571577 \\
\hline Taiji Group & 0.67919953 & 0.452980777 & 0.474001541 & 0.387892653 \\
\hline Taloph Pharm & 0.842636824 & 0.438224781 & 0.689161525 & 0.607715363 \\
\hline Teyi & 0.836358694 & 0.453682077 & 0.749014352 & 0.629612238 \\
\hline
\end{tabular}


TABle 4: Continued.

\begin{tabular}{|c|c|c|c|c|}
\hline \multirow{2}{*}{ Company name } & \multicolumn{4}{|c|}{ Year } \\
\hline & 2015-2016 & 2016-2017 & $2017-2018$ & 2018-2019 \\
\hline Tasly & 0.717299163 & 0.615735065 & 0.675224149 & 0.512843572 \\
\hline Tiantan Biological & 0.678613559 & 0.591061254 & 0.860246774 & 0.500479724 \\
\hline Tianyao Pharm & 0.857677116 & 0.448393948 & 0.737240947 & 0.409261416 \\
\hline Thdb & 0.900688131 & 0.545722415 & 0.888741777 & 0.491006733 \\
\hline Thjm & 0.744976147 & 0.47311369 & 0.785334315 & 0.254122817 \\
\hline TRT & 0.836177972 & 0.656151609 & 0.703560723 & 0.570595311 \\
\hline Wanbangde & 0.792851861 & 0.453600664 & 0.482306406 & 0.423211786 \\
\hline Wondfo & 0.718234154 & 0.423654128 & 0.712394655 & 0.672912535 \\
\hline WEDGE INDUSTRIAL & 0.78623338 & 0.450705667 & 0.727173666 & 0.609309043 \\
\hline Weiming & 0.801846721 & 0.489019559 & 0.697530662 & 0.612890373 \\
\hline Wowu & 0.915178281 & 0.444228865 & 0.732530156 & 0.611424148 \\
\hline Wohua & 0.849951067 & 0.451158165 & 0.726657243 & 0.616986053 \\
\hline Wosen & 0.293538834 & 0.36896297 & 0.927581915 & 0.621532855 \\
\hline AMD & 0.565789697 & 0.46605309 & 0.755941006 & 0.654308568 \\
\hline Xianju Pharm & 0.722129826 & 0.46975224 & 0.788610822 & 0.449576159 \\
\hline Xiangxue Pharm & 0.56594413 & 0.450246609 & 0.728601595 & 0.406168036 \\
\hline Sunflower & 0.775727247 & 0.435119495 & 0.472627581 & 0.583260445 \\
\hline NHU & 0.744446192 & 0.084792409 & 0.174517515 & 0.645247085 \\
\hline Xinhua & 0.686150026 & 0.418580131 & 0.691475644 & 0.430862405 \\
\hline Xinbang & 0.37146641 & 0.482379256 & 0.29706599 & 0.42940549 \\
\hline SALUBRIS & 0.828950689 & 0.45514634 & 0.744067755 & 0.492886897 \\
\hline BROTHER & 0.867361567 & 0.491290966 & 0.722969193 & 0.404592688 \\
\hline Yabao & 0.62538223 & 0.465981424 & 0.776164146 & 0.400865973 \\
\hline Yatai & 0.791869911 & 0.458579905 & 0.749488501 & 0.274754037 \\
\hline Yananbikang & 0.609592291 & 0.525754839 & 0.495889903 & 0.382123694 \\
\hline Yiling Pharm & 0.732199742 & 0.505748671 & 0.83520904 & 0.467694136 \\
\hline Yifan & 0.895380366 & 0.428163195 & 0.614491215 & 0.498168738 \\
\hline Yibai & 0.622820274 & 0.32775112 & 0.386250987 & 0.412500415 \\
\hline Yisheng & 0.615818561 & 0.450233859 & 0.733856106 & 0.615039599 \\
\hline Yiduoli & 0.768370215 & 0.454151698 & 0.749092834 & 0.418880633 \\
\hline Chinataurine & 0.790572254 & 0.445496958 & 0.734787151 & 0.618625291 \\
\hline Gloria Pharm & 0.425375138 & 0.473021826 & 0.735198757 & 0.099279291 \\
\hline Baiyao & 0.676420897 & 0.831225519 & 0.898534261 & 0.870529199 \\
\hline Zhejiang Medicine & 0.648977621 & 0.470928238 & 0.508877262 & 0.43259115 \\
\hline Zhenbao Island & 0.366714214 & 0.44796952 & 0.719005835 & 0.445442437 \\
\hline Zdzy & 0.666462777 & 0.477272075 & 0.688004343 & 0.415110578 \\
\hline Zhifei & 0.794312561 & 0.48858374 & 1 & 0.851446092 \\
\hline Zhongguancun & 0.638431487 & 0.361048475 & 0.600829723 & 0.58735967 \\
\hline China Medicine & 0.600385661 & 0.632935767 & 0.705012728 & 0.541274783 \\
\hline Zhongheng Group & 0.779488337 & 0.516431725 & 0.842980772 & 0.726827094 \\
\hline Zhongmu & 0.891791426 & 0.461595881 & 0.758629587 & 0.388861314 \\
\hline Zhongxin & 0.784299166 & 0.500035996 & 0.833734288 & 0.470157549 \\
\hline Zsyy & 0.712236556 & 0.493776655 & 0.805881833 & 0.435730452 \\
\hline JLZX & 0.56839467 & 0.487351822 & 0.7538412 & 0.407572713 \\
\hline Zuoli & 0.506830635 & 0.327842837 & 0.530045226 & 0.603856046 \\
\hline
\end{tabular}


TABLe 5: Analysis of the overall efficiency of Chinese pharmaceutical companies.

\begin{tabular}{|c|c|c|c|c|}
\hline \multirow{2}{*}{ Company name } & \multicolumn{4}{|c|}{ Year } \\
\hline & $2015-2016$ & 2016-2017 & $2017-2018$ & 2018-2019 \\
\hline Adisseo & 1 & 0.594331961 & 0.687253527 & 0.590582307 \\
\hline Anke Bio & 0.732050473 & 0.603904778 & 0.721684173 & 0.658510045 \\
\hline Osaikon & 0.778427713 & 0.599091917 & 0.832059909 & 0.696276311 \\
\hline Baiyunshan & 0.228285921 & 0.347396666 & 0.496661228 & 0.354314544 \\
\hline Bdyy & 0.740297813 & 0.537030807 & 0.6599985 & 0.641144699 \\
\hline Beilu & 0.754848982 & 0.608873049 & 0.726868379 & 0.745366839 \\
\hline Porton & 0.704058135 & 0.537439838 & 0.673751419 & 0.645425288 \\
\hline None & 0.804540469 & 0.581032222 & 0.686403127 & 0.634019398 \\
\hline Changchun High-Tech & 0.670785268 & 0.613981329 & 0.826328221 & 0.91902694 \\
\hline Changjiang Runfa Medicine & 0.709676062 & 0.545533564 & 0.667403235 & 0.486189125 \\
\hline Changshan Pharma & 0.771002674 & 0.594856424 & 0.68892317 & 0.682407653 \\
\hline DAJY & 0.553391996 & 0.532804722 & 0.650855774 & 0.625961732 \\
\hline Dezhan & 0.889124007 & 0.713057908 & 0.85424928 & 0.696289729 \\
\hline Jiao & 0.847938007 & 0.724106276 & 0.892313767 & 0.434427484 \\
\hline DBBT & 0.745018596 & 0.588398867 & 0.704618016 & 0.676626886 \\
\hline $\mathrm{VC}$ & 0.4392569 & 0.411082825 & 0.544473127 & 0.465493745 \\
\hline Dongcheng & 0.780465431 & 0.58515561 & 0.710439698 & 0.633026364 \\
\hline Nhwa Pharm & 0.734368139 & 0.53849531 & 0.686067979 & 0.687237305 \\
\hline Ekzy & 0.679929415 & 0.567141171 & 0.651349898 & 0.617078583 \\
\hline Fangsheng & 0.763857057 & 0.576574634 & 0.69884161 & 0.674382377 \\
\hline Fengyuan & 0.444827414 & 0.480815113 & 0.614783892 & 0.606094921 \\
\hline Fczy & 0.742074088 & 0.578441415 & 0.685449982 & 0.654076242 \\
\hline Fayy & 0.747025125 & 0.542767359 & 0.550777941 & 0.641860364 \\
\hline Fosun Pharm & 0.423354375 & 0.670393153 & 0.67734337 & 0.657568579 \\
\hline Fuxiang & 0.690619828 & 0.558173035 & 0.657502803 & 0.69283151 \\
\hline Guangji & 0.723829795 & 0.562163776 & 0.697307481 & 0.671209547 \\
\hline Kwong Sang Hong & 0.782406423 & 0.601677149 & 0.712736266 & 0.684833649 \\
\hline Guang Yuyuan & 0.78963604 & 0.597988236 & 0.731277172 & 0.563852758 \\
\hline Lark & 0.724214801 & 0.557523041 & 0.691343122 & 0.603175409 \\
\hline Glsj & 0.704470405 & 0.600924412 & 0.722522094 & 0.691917544 \\
\hline Sinopharm Hyundai & 0.427413377 & 0.393573592 & 0.554655968 & 0.411827436 \\
\hline Harbin Pharm & 0.352405918 & 0.369339239 & 0.485586707 & 0.432828982 \\
\hline Haili Bio & 0.783470209 & 0.604343839 & 0.699572898 & 0.67374349 \\
\hline Hnhy & 0.569927767 & 0.533436376 & 0.636600922 & 0.537164476 \\
\hline Hepalink & 0.592158759 & 0.525343452 & 0.699393429 & 0.659703397 \\
\hline Haishun New Pharma & 0.8030248 & 0.605941868 & 0.716134725 & 0.700106885 \\
\hline Haisco & 0.480099239 & 0.575867685 & 0.699064728 & 0.661359713 \\
\hline Hisoar & 0.671469103 & 0.541039094 & 0.710909693 & 0.676879776 \\
\hline Haixin & 0.734260582 & 0.551448437 & 0.676486256 & 0.637198392 \\
\hline Hisun & 0.287396529 & 0.380594016 & 0.415821467 & 0.441140083 \\
\hline Han Sen Pharm & 0.732716709 & 0.559696588 & 0.688766917 & 0.684174582 \\
\hline Hybio & 0.724090442 & 0.622127582 & 0.609611185 & 0.477857438 \\
\hline Hengrui & 0.826957748 & 0.770628784 & 1 & 1 \\
\hline Chase Sun & 0.550775748 & 0.399677749 & 0.436619602 & 0.528426739 \\
\hline NCPC & 0.354218369 & 0.358094194 & 0.480775578 & 0.427708854 \\
\hline Huahai & 0.497285595 & 0.525399389 & 0.58350641 & 0.588866754 \\
\hline Hualan Bio & 0.606253871 & 0.611156906 & 0.786676493 & 0.803468984 \\
\hline Huaren & 0.703067183 & 0.5262557 & 0.649580709 & 0.631043029 \\
\hline HRSJ & 0.406183354 & 0.51553843 & 0.651086789 & 0.659592625 \\
\hline China Resources Double-Crane & 0.464181045 & 0.478110469 & 0.667487244 & 0.618744398 \\
\hline Huashen Technology & 0.79585331 & 0.605253566 & 0.698514176 & 0.686848921 \\
\hline Walter Dyne & 0.745780253 & 0.592281438 & 0.685737585 & 0.679786234 \\
\hline Yanbian FC & 0.58552906 & 0.726843818 & 0.75539478 & 0.760336119 \\
\hline Kyrgyzstan & 0.57291471 & 0.595278792 & 0.719807265 & 0.307289038 \\
\hline Jichuan & 0.673524741 & 0.478385974 & 0.569904186 & 0.621509323 \\
\hline Jimin & 0.755191647 & 0.581216669 & 0.682498022 & 0.658109286 \\
\hline JYPC & 0.789213967 & 0.533152421 & 0.692664421 & 0.655377456 \\
\hline Joincare Pharm & 0.373389357 & 0.612787948 & 0.431248192 & 0.711024008 \\
\hline Jiangzhong & 0.773280624 & 0.57457233 & 0.714383571 & 0.696034497 \\
\hline
\end{tabular}


TABle 5: Continued.

\begin{tabular}{|c|c|c|c|c|}
\hline \multirow{2}{*}{ Company name } & \multicolumn{4}{|c|}{ Year } \\
\hline & $2015-2016$ & 2016-2017 & $2017-2018$ & 2018-2019 \\
\hline Jincheng & 0.6424688 & 0.541255874 & 0.633395756 & 0.600807083 \\
\hline Jinhe Bio & 0.694022214 & 0.53855834 & 0.672530294 & 0.639655629 \\
\hline Jinling & 0.669922726 & 0.463558983 & 0.63122491 & 0.598918507 \\
\hline Jinshiya & 0.779202218 & 0.61261129 & 0.67505169 & 0.654243898 \\
\hline Jingxin & 0.684642416 & 0.522548631 & 0.634930264 & 0.670080794 \\
\hline Jinghua & 0.73659423 & 0.580261028 & 0.708115153 & 0.532218118 \\
\hline Jingfeng & 0.657549858 & 0.543871346 & 0.658966242 & 0.439651462 \\
\hline Jiuqiang & 0.832168543 & 0.640440908 & 0.760447378 & 0.740948042 \\
\hline Jiuzhitang & 0.660685922 & 0.510365495 & 0.551518743 & 0.601037942 \\
\hline Jiuzhou & 0.615906479 & 0.511229588 & 0.651701562 & 0.646607443 \\
\hline CONBA & 0.43789997 & 0.490049729 & 0.667671711 & 0.454110037 \\
\hline Kanghong & 0.640270409 & 0.530393868 & 0.625355868 & 0.589391549 \\
\hline Kangyuan & 0.558531561 & 0.511112483 & 0.661025134 & 0.653468887 \\
\hline Kangzhi & 0.770954193 & 0.582966967 & 0.690695416 & 0.63784734 \\
\hline KHB & 0.719324825 & 0.576138757 & 0.689042667 & 0.655129362 \\
\hline Kelun & 0.350816311 & 0.399255114 & 0.527654704 & 0.466336149 \\
\hline Sunflower & 0.450269017 & 0.47246729 & 0.609455977 & 0.602948399 \\
\hline Kunming Pharm & 0.486232652 & 0.488722513 & 0.622323739 & 0.575298309 \\
\hline Lummy & 0.676182846 & 0.548086309 & 0.67791945 & 0.60414936 \\
\hline LAYN & 0.766415526 & 0.615493713 & 0.696786083 & 0.687666604 \\
\hline Lisheng Pharma & 0.772324056 & 0.553232118 & 0.688792627 & 0.658713187 \\
\hline Livzon Pharm & 0.406802204 & 0.950736975 & 0.693561727 & 0.641403963 \\
\hline LEADMAN & 0.7148845 & 0.589683903 & 0.697033474 & 0.677283392 \\
\hline Lianhuan Pharm & 0.777990479 & 0.580819977 & 0.70187494 & 0.677825662 \\
\hline Lingkang & 0.593122777 & 0.602274773 & 0.726097851 & 0.699381382 \\
\hline Lingrui Pharm & 0.747485965 & 0.570748655 & 0.691654705 & 0.678247727 \\
\hline Long Jin Pharm & 0.778557308 & 0.60796337 & 0.720057661 & 0.694828539 \\
\hline Lukang Pharm & 0.568388015 & 0.368837292 & 0.454369366 & 0.548391093 \\
\hline Mike Bio & 0.768849987 & 0.607581032 & 0.730833097 & 0.678396297 \\
\hline M.k. & 0.705468047 & 0.594140747 & 0.659621157 & 0.473923489 \\
\hline Palin Bio & 0.735879001 & 0.580588028 & 0.7000859 & 0.692170464 \\
\hline PIEN TZE HUANG & 0.794732455 & 0.644348252 & 0.804287685 & 0.736290866 \\
\hline Julie Plec & 0.628612707 & 0.51441293 & 0.586701026 & 0.629100125 \\
\hline Plyy & 0.470181321 & 0.418413253 & 0.567877026 & 0.583837057 \\
\hline CHEEZHENGTTM & 0.625915805 & 0.595282402 & 0.719854866 & 0.715491847 \\
\hline Qidi & 0.763992733 & 0.561948196 & 0.677246094 & 0.688875522 \\
\hline Qianhong Biopharma & 0.796996434 & 0.587850281 & 0.703561836 & 0.674562645 \\
\hline Qianjin Pharm & 0.634951449 & 0.510221787 & 0.651319311 & 0.643983464 \\
\hline Qianyuan & 0.734922726 & 0.538059322 & 0.641532464 & 0.529123309 \\
\hline Renfu & 0.334316504 & 0.515659371 & 0.162643485 & 0.472524591 \\
\hline Renhe Pharmacy & 0.710690176 & 0.501475944 & 0.678520156 & 0.647375907 \\
\hline Rpsw & 0.616216798 & 0.544427634 & 0.663887992 & 0.660390626 \\
\hline Saisheng & 0.842078163 & 0.636453134 & 0.751848255 & 0.697189691 \\
\hline SAM & 0.793087056 & 0.570374323 & 0.637288238 & 0.66250408 \\
\hline Shanhe Pharmacy & 0.780593912 & 0.590341994 & 0.705650192 & 0.693438972 \\
\hline Shkb & 0.769351682 & 0.596263047 & 0.707135607 & 0.684164457 \\
\hline Shanghai RAAS Blood Products & 0.907968608 & 0.628170623 & 0.352321342 & 0.675478039 \\
\hline Shenqi & 0.680638994 & 0.523233731 & 0.611259379 & 0.641985589 \\
\hline Biological Stock & 0.870829861 & 0.70347757 & 0.801965497 & 0.656218882 \\
\hline Salvage Pharm & 0.547394207 & 0.443227806 & 0.638664943 & 0.301830016 \\
\hline Sts & 0.840550192 & 0.630975455 & 0.719756982 & 0.666578994 \\
\hline Scyy & 0.680239263 & 0.581555378 & 0.673278766 & 0.671620779 \\
\hline Beijing SL Pharm & 0.744354754 & 0.664713516 & 0.785954743 & 0.730167136 \\
\hline Stellite & 0.725188753 & 0.558580997 & 0.681979752 & 0.655174892 \\
\hline Shsw & 0.798450652 & 0.591904871 & 0.700994849 & 0.696898497 \\
\hline Tat & 0.712944165 & 0.470305378 & 0.557717995 & 0.553727441 \\
\hline Taiji Group & 0.523948741 & 0.370677112 & 0.489754105 & 0.411216635 \\
\hline Taloph Pharm & 0.723845517 & 0.541466331 & 0.629307653 & 0.632734845 \\
\hline Teyi & 0.760999041 & 0.581909561 & 0.708162034 & 0.680597811 \\
\hline
\end{tabular}


Table 5: Continued.

\begin{tabular}{|c|c|c|c|c|}
\hline \multirow{2}{*}{ Company name } & \multicolumn{4}{|c|}{ Year } \\
\hline & 2015-2016 & 2016-2017 & 2017-2018 & 2018-2019 \\
\hline Tasly & 0.531017743 & 0.490066656 & 0.611366055 & 0.461437582 \\
\hline Tiantan Biological & 0.622734244 & 0.66310047 & 0.782922006 & 0.712465273 \\
\hline Tianyao Pharm & 0.712730596 & 0.544247373 & 0.652803637 & 0.608985283 \\
\hline Thdb & 0.795554142 & 0.653229828 & 0.790400206 & 0.731561874 \\
\hline Thjm & 0.722867728 & 0.577589877 & 0.703162547 & 0.260771659 \\
\hline TRT & 0.643059805 & 0.554356733 & 0.641063018 & 0.544020294 \\
\hline Wanbangde & 0.571625007 & 0.432249488 & 0.473426951 & 0.453497617 \\
\hline Wondfo & 0.712134043 & 0.586408694 & 0.689386071 & 0.705688037 \\
\hline WEDGE INDUSTRIAL & 0.75213728 & 0.609551683 & 0.708716889 & 0.688673642 \\
\hline Weiming & 0.775011865 & 0.62907394 & 0.658692074 & 0.661517811 \\
\hline Wowu & 0.827238366 & 0.614236394 & 0.735571328 & 0.715768679 \\
\hline Wohua & 0.762137003 & 0.580657605 & 0.692834657 & 0.694553785 \\
\hline Wosen & 0.456421123 & 0.470227422 & 0.862487868 & 0.660496829 \\
\hline AMD & 0.627878133 & 0.627997177 & 0.734618138 & 0.732444295 \\
\hline Xianju Pharm & 0.618297341 & 0.518121431 & 0.664360204 & 0.633863302 \\
\hline Xiangxue Pharm & 0.551863899 & 0.495157616 & 0.604938414 & 0.560265277 \\
\hline Sunflower & 0.657878385 & 0.517059063 & 0.430579818 & 0.644671298 \\
\hline NHU & 0.631069124 & 0.344337963 & 0.417527717 & 0.696876755 \\
\hline Xinhua & 0.544129945 & 0.424368226 & 0.564325286 & 0.5586054 \\
\hline Xinbang & 0.400791325 & 0.455593724 & 0.329151623 & 0.485028396 \\
\hline SALUBRIS & 0.786916152 & 0.617220238 & 0.720859622 & 0.566009985 \\
\hline BROTHER & 0.76537271 & 0.605409971 & 0.653283686 & 0.614230582 \\
\hline Yabao & 0.539400468 & 0.486947908 & 0.645626561 & 0.578342875 \\
\hline Yatai & 0.739747264 & 0.591038926 & 0.704074772 & 0.291857375 \\
\hline Yananbikang & 0.612340283 & 0.562211409 & 0.565493676 & 0.436795179 \\
\hline Yiling Pharm & 0.633799819 & 0.526020841 & 0.678106867 & 0.616128252 \\
\hline Yifan & 0.758376423 & 0.567154214 & 0.578959385 & 0.633371792 \\
\hline Yibai & 0.612320129 & 0.455498536 & 0.365362945 & 0.569666434 \\
\hline Yisheng & 0.628263309 & 0.564718437 & 0.678697744 & 0.659088427 \\
\hline Yiduoli & 0.693578342 & 0.527624776 & 0.658030562 & 0.612212776 \\
\hline Chinataurine & 0.722247367 & 0.580681117 & 0.704650362 & 0.672924197 \\
\hline Gloria Pharm & 0.5225888 & 0.528091646 & 0.634974266 & 0.140262719 \\
\hline Baiyao & 0.542062095 & 0.623275676 & 0.74860877 & 0.731659557 \\
\hline Zhejiang Medicine & 0.522959555 & 0.432463484 & 0.56815758 & 0.508852734 \\
\hline Zhenbao Island & 0.491175612 & 0.539218332 & 0.642235011 & 0.646298006 \\
\hline Zdzy & 0.602884228 & 0.509387255 & 0.567643001 & 0.581329044 \\
\hline Zhifei & 0.726661912 & 0.633983832 & 0.922890095 & 0.925723046 \\
\hline Zhongguancun & 0.623208215 & 0.479354766 & 0.58000983 & 0.617714904 \\
\hline China Medicine & 0.44933969 & 0.458628776 & 0.547204643 & 0.43667571 \\
\hline Zhongheng Group & 0.718912618 & 0.623627731 & 0.751932612 & 0.737063988 \\
\hline Zhongmu & 0.679383423 & 0.483648804 & 0.619691314 & 0.535837661 \\
\hline Zhongxin & 0.605084581 & 0.489320281 & 0.654535316 & 0.592485773 \\
\hline Zsyy & 0.692260649 & 0.607808063 & 0.723569987 & 0.656790838 \\
\hline JLZX & 0.613219972 & 0.606033666 & 0.675253694 & 0.61472573 \\
\hline Zuoli & 0.576940662 & 0.497474779 & 0.573208842 & 0.653638646 \\
\hline
\end{tabular}

Table 6: Annual Malmquist index and its decomposition indexes.

\begin{tabular}{lccccc}
\hline & EC & SC & TC & PC & TFP \\
\hline $2015-2016$ & 1.1841 & 2.7910 & 1.0109 & 0.4242 & 0.3567 \\
$2016-2017$ & 0.5162 & 1.4472 & 1.1211 & 0.3978 & 0.5787 \\
$2017-2018$ & 0.9815 & 2.4676 & 1.0018 & 0.4260 & 0.9833 \\
$2018-2019$ & 0.5188 & 1.2176 & 1.0034 & 0.5205 \\
Mean & 0.8001 & 1.9808 & 1.0343 & 0.4012 & 0.8219 \\
\hline
\end{tabular}




\section{Dynamic Effect Analysis}

The efficiency of the two-stage network DEA model varies from year to year, and the efficiency value of different years is not comparable, so time series analysis cannot be carried out. To make up for the shortcomings of the traditional twostage network DEA, this paper adds the Malmquist index to study the total factor productivity of listed pharmaceutical companies in 2015-2019 and quantify its decomposition limit. The results are shown in Table 6.

The average total factor productivity (TFP) is 0.8219 that has fallen by an average $17.81 \%$. Viewed from the decomposition index, the mean of EC is 0.8001 ; that is, EC has decreased by an average of $19.99 \%$. The mean of PC is 0.4012 , with the rate of decline in each averaging over $59.88 \%$ a year. SC is 1.9808 , with an average annual growth rate of $98.08 \%$. It shows that the operating efficiency of listed pharmaceutical enterprises depends on the scale expansion and makes up for low management efficiency. The average technology change (TC) is 1.0343 , and it has risen by nearly $3.43 \%$ per year. The technology change has been improved between 2015 and 2019.

The listed pharmaceutical companies rely on product development and can be combined with innovative technologies. For the pharmaceutical industry, the level of research and development of products indirectly affects the level of industry development. The drugs or pharmaceutical equipment is very important; if the level of medical technology research and development is not advanced enough, the progress of medical level will be affected. Therefore, the listed pharmaceutical enterprises should rely on the existing advanced technology achievements, improving their own technology, to improve operating efficiency.

\section{Conclusion}

This paper firstly divides the two subsystems by using the two-stage network DEA and analyses the operating efficiency of 1,63 listed pharmaceutical companies in China from 2015 to 2019. Secondly, Malmquist index is used for dynamic analysis; the total factor productivity and decomposition limit were obtained. Finally, we make some suggestions based on the results of the study.

From the results, the technical efficiency of the second phase is less than that of the first stage; government subsidies have no positive impact on most companies. It is possible that enterprises move government subsidies elsewhere rather than pharmaceutical companies. It is also possible that government subsidies have increased, enterprises are more willing to invest in product development and enterprises expansion, and it is difficult to see the improvement of operational efficiency in the short term. However, the government subsidies have a positive impact on enterprises with low efficiency in a short term. To ensure the efficiency of investment and avoid waste of resources, government needs to choose the object of subsidies carefully and reformulate policies that encourage pharmaceutical listed companies. And according to the Malmquist index results, enterprises should pay attention to risk prevention and avoid rapid expansion bringing in diseconomies of scale. All in all, enterprises should improve management level and technological capabilities and shift scale growth to total factor productivity.

However, there are also some limitations. Regarding the data resources, the data we chose cannot exactly predict the operational situations among current Chinese medical firms, since there is more uncertainty in the market, especially during the COVID-19 period, which is likely to be a potential direction that other scholars can study further in the future. Concerning variables, this article analyses the operational efficiency of 164 firms; researchers can only choose several companies to make an in-depth analysis instead of the whole industrial analysis.

\section{Data Availability}

The data used to support the findings of this study are available from the corresponding author upon request.

\section{Conflicts of Interest}

The authors declare there are no conflicts of interest regarding the publication of this paper.

\section{Acknowledgments}

This research was financially supported by Guangdong Planning Office of Philosophy and Social Sciences Project (Youth): Research on cross-border social responsibility of private foreign trade enterprises in Guangdong and the reconstruction of legitimacy-Perspective of the organization to piece together. (Project number: GD20YGL09). And it also supported by Department of Education of Guangdong Province "Innovative projects with characteristics of ordinary universities" project: Research on Sustainable Development of Foreign Trade in Guangdong Province Based on Energy Footprint (Project no. 2019WTSCX158). Moreover, it is also supported by Key Discipline-International Business Construction and Development Project (Project no. HS2019CXQX17).

\section{References}

[1] Y. Wang, "Research on the financing efficiency of biomedicine industry based on DEA model," Hebei Enterprise, no. 01, pp. 104-105, 2021.

[2] Z. Zhang, Research on Performance Evaluation of Pharmaceutical Enterprises Based on AHP-DEA Method, Tianjin University, Tianjin, China, 2018.

[3] Z. Li, R. Liu, H. He, and J. Qin, "An empirical study on the efficiency of the pharmaceutical industry based on the SEDEA model," Accounting Communications, no. 14, pp. 26-28, 2016.

[4] X. Zhou and C. Luo, "Research on the relative effectiveness of China's pharmaceutical industry technological innovation based on DEA model," Science and Technology Management Research, no. 9, pp. 252-254, 2009.

[5] P. Shi and L. Tao, "Evaluation of innovation performance of pharmaceutical industry based on DEA model," Economic Perspective, no. 3, pp. 28-29, 2011. 
[6] H. Chen, Research on Technology Innovation Efficiency of China's Pharmaceutical Industry-Analysis Based on Panel Data, Jinan University, Guangzhou, China, 2011.

[7] J. Liu, "Study on performance evaluation of science and technology investment in China's pharmaceutical high-tech industry," Science Management Research, vol. 27, no. 4, pp. 35-38, 2009.

[8] A. Xiong, Research on Technological Innovation Efficiency of Pharmaceutical Companies Based on DEA Method, Guangdong Pharmaceutical University, Guangzhou, China, 2020.

[9] H. F. Lewis, S. Mallikarjun, and T. R. Sexton, "Unoriented two-stage DEA: the case of the oscillating intermediate products," European Journal of Operational Research, vol. 229, no. 2, pp. 529-539, 2013.

[10] L. Liang, Z.-Q. Li, W. D. Cook et al., "Data envelopment analysis efficiency in two-stage networks with feedback," IIE Transactions, vol. 43, no. 5, pp. 309-322, 2010.

[11] L. Liang, D. Wade, and J. Z. Cook, "DEA models for two-stage processes: game approach and efficiency decomposition," Naval Research Logistics, vol. 55, no. 7, pp. 643-653, 2008.

[12] Y. Chen, J. Du, H. David Sherman et al., "DEA model with shared resources and efficiency decomposition," European Journal of Operational Research, vol. 207, no. 1, pp. 339-349, 2010.

[13] C. Kao, "Efficiency decomposition in network data envelopment analysis with slacks-based measures," European Journal of Operational Research, vol. 192, no. 3, pp. 949-962, 2009.

[14] C.-Y. Lee, L. Andrew, and Z. Johnson, "A decomposition of productivity change in the semiconductor manufacturing industry," International Journal of Production Research, vol. 49, no. 16, pp. 4761-4785, 2011.

[15] W. T. Pan, M. E. Zhuang, Y. Y. Zhou et al., "Research on sustainable development and efficiency of China's E-agriculture based on a data envelopment analysis-malmquist model," Technological Forecasting and Social Change, vol. 162, Article ID 120298, 2021.

[16] A. Hashimoto and S. Haneda, "Measuring the change in R\&D efficiency of the Japanese pharmaceutical industry," Research Policy, vol. 37, no. 10, pp. 1829-1836, 2008.

[17] H. Pannu, U. Kumar, and J. Farooqui, Impact of Innovationon the Performance of Indian Pharmaceutical Industry Using Data Envelopment Analysis, IIMB working paper, Karnataka, India, 2010.

[18] X. Zhiyue and J. Qiu, "Evaluation and analysis of the operating efficiency of my country's biopharmaceutical industry," Shanghai Medicine, vol. 36, no. 13, pp. 50-53, 2015.

[19] P. Cheng and C. Zheng, "Research on my country's science and technology input efficiency based on decision preference two-stage network DEA," Science and Technology Progress and Policy, vol. 31, no. 08, pp. 125-129, 2014. 